\title{
Biological Activities of Lignoids from Amazon Myristicaceae Species: Virola michelii, V. mollissima, V. pavonis and Iryanthera juruensis"
}

\author{
Sabrina K. R. Morais, ${ }^{a, b}$ Ana F. Teixeira, ${ }^{a, c}$ Zelina E. dos S. Torres, ${ }^{d, e}$ Sergio M. Nunomura, ${ }^{e}$ \\ Edite H. Yamashiro-Kanashiro, ${ }^{f}$ José Angelo L. Lindoso ${ }^{f}$ and Massayoshi Yoshida ${ }^{*, a, b}$
}

aInstituto de Química, Universidade de São Paulo, Av. Prof. Lineu Prestes, 748, 05508-900 São Paulo-SP, Brazil

${ }^{b}$ Centro de Biotecnologia da Amazônia, Av. Gov. Danilo de Matos Areosa, 690, 69075-351 Manaus-AM, Brazil

${ }^{c}$ Escola Normal Superior, Universidade do Estado do Amazonas, Av. Djalma Batista, 2470, 69050-010 Manaus-AM, Brazil

dEscola Superior de Ciências da Saúde, Universidade do Estado do Amazonas, Av. Carvalho Leal, 1777, 69065-001 Manaus-AM, Brazil

${ }^{e}$ Coordenação de Pesquisas de Produtos Naturais, Instituto Nacional de Pesquisas da Amazônia, Av. André Araujo, 2936, 69060-001 Manaus-AM, Brazil

${ }^{f}$ Laboratório de Soroepidemiologia (LIM-38 HC-FMUSP) e Instituto de Infectologia Emilio Ribas SES-SP, São Paulo-SP, Brazil

\begin{abstract}
O trabalho consiste na re-investigação dos frutos de Iryanthera juruensis e Virola pavonis e das folhas de $V$. michelii, bem como no estudo dos frutos de V. mollissima. A partir de I. juruensis foram isoladas uma neolignana ariltetralínica (1) e uma neolignana tetraidrofurânica (2), enquanto que de Virola pavonis foram isoladas neolignanas benzofurânicas (6-9), tetraidrofurânicas (2, 11-13, 17), bifenílica (10), diastereoisômeros de uma hidróxi-neolignana 8.O.4'(14-15) e outras. $V$. mollissima acumula a neolignana ariltetralônica $\mathbf{4}$ ou o seu derivado seco (5). As folhas de $V$. michelii apresentaram a ocorrência de lignanas furofurânicas (18-19). Os lignóides $\mathbf{1}$ e $\mathbf{2}$ obtidos dos arilos de I. juruensis apresentaram atividade leishmanicida contra a forma promastigota de Leishmania amazonensis.
\end{abstract}

This work revisits the fruits of Iryanthera juruensis and Virola pavonis and the leaves from $V$. michelii, as well as describing a study of the fruits of V. mollissima. In I. juruensis aryltetraline neolignan (1) and tetrahydrofuran neolignan (2), were found while from V. pavonis neolignans of benzofuran type (6-9), the tetrahydrofuran type $(\mathbf{2}, 11-13,17)$ and the biphenyl type $(10)$, in addition to diastereoisomers of the 8.O.4'-oxyneolignan type (14 and 15) and others were isolated. The V. mollissima accumulates the aryltetralone neolignan $\mathbf{4}$ and its seco derivative (5). The lignoids $\mathbf{1}$ and $\mathbf{2}$ obtained from I. juruensis arils possess antileishmanial activity against the promastigote form of Leishmania amazonensis.

Keywords: lignoids, Iryanthera juruensis, Virola michelii, Virola mollissima, Virola pavonis, Myristicaceae

\section{Introduction}

Myristicaceae is a botanical family composed of 18 genera and about 500 species with pantropical

*e-mail: myoshida@iq.usp.br

\# This paper is dedicated to Prof. Otto R. Gottlieb, who made an important phytochemical contribution to plant species from the Amazon rain forest, describing the occurrence of neolignans in Lauraceae and Myristicaceae species (Gottlieb, O. R.; Yoshida, M.; Lignans, in Natural Products of Woody Plants: Chemicals Extraneous to the Lignocellulosic Cell Wall, J.W. Rowe, ed., Springer-Verlag, Berlin, 1989, ch. 7.3, pp. 439-511). distribution. The species are normally trees, exceptionally shrubs, frequently found in lowland forests. In Brazil, this family is represented by the genera Compsoneura, Iryanthera, Osteophloeum, Otoba (syn. Dialyanthera) and Virola, which are concentrated in the Amazon region, where the myristicaceous species are popularly known as "ucuúba".1,2

The species belonging to this family became the target of intense study when Schultes reported that some indigenous tribes of the Amazon used the barks and resins 
of Virola spp. for the preparation of hallucinogenic snuffs and arrow poisons for hunting. ${ }^{1}$

The phytochemical investigations on myristicaceous species have shown the accumulation of a variety of lignoids in their tissues, which have relevant pharmacological activities that are well documented in the literature. ${ }^{3,4}$ While there are several studies about the chemical composition of Amazon Myristicaceae species, the biological activities of these species could be better studied. This paper aims to contribute to the chemosystematics of Myristicaceae species and to assay some biological activities in vitro, by reporting a phytochemical re-investigation of the fruits from Iryanthera juruensis and Virola pavonis and of the leaves from Virola michelii, as well as a new study of the fruits of Virola mollissima as well as the results of biological assays of isolated lignoids.

\section{Experimental}

General

${ }^{1} \mathrm{H}$ and ${ }^{13} \mathrm{C}$ NMR spectra were recorded on spectrometers from Bruker AC-200 and Varian INOVA 200, 4.7 T, operating at $200 \mathrm{MHz}$ and $50 \mathrm{MHz}$, respectively, Varian INOVA 300, 7.4 T, operating at $300 \mathrm{MHz}$ and $75 \mathrm{MHz}$, respectively, and Varian INOVA 500, 11.7 T, operating at $500 \mathrm{MHz}$ and $125 \mathrm{MHz}$, respectively. The samples were dissolved in $\mathrm{CDCl}_{3}$ or $\mathrm{CD}_{3} \mathrm{CN}$, purchased from Aldrich or CIL.

\section{Plant material}

About $1.0 \mathrm{~kg}$ of ripe fruits from I. juruensis Warburg were collected on the campus of the Universidade Federal do Amazonas (March 2003), in Manaus-AM, while the fruits of V. mollissima (Poepp. ex A. DC.) Warburg and V. pavonis (A. DC.) A.C. Smith were obtained from the Adolpho Ducke Reserve, Manaus-AM, in November 2003, and March 2004, respectively. The botanical materials collected were identified by José Ferreira Ramos. Virola michelii Heckel leaves were collected on Campus I of the Instituto Nacional de Pesquisas da Amazônia (INPA), Manaus-AM, in December 2004. Vouchers were deposited at the INPA herbarium under the numbers: 213356 (I.j.), 179760 (V.mo.), 214917 (V.p.) and 215688 (V.mi.).

\section{Extraction and isolation}

The ripe fruits (I. juruensis, V. mollissima and V. pavonis) were manually separated into pericarps and seeds and the latter into arils, seed coats and almonds, which were dried, milled and macerated individually with hexane followed by EtOH, during a total period of fourteen days. After the extractions, the solutions were concentrated under reduced pressure yielding their hexane and EtOH extracts. The crude hexane extracts were dissolved in 9:1 MeOH:water, and submitted to partition with hexane, yielding the hexane and hydromethanolic fractions. Similarly, the crude $\mathrm{EtOH}$ extracts were partitioned with hexane, $\mathrm{CHCl}_{3}$ and EtOAc, generating four additional fractions.

The $\mathrm{CHCl}_{3}$ residue of the EtOH extract from $I$. juruensis arils (960.0 mg) was submitted to column chromatography on silica gel eluting with hexane:EtOAc in mixtures of increasing polarity, affording 20 fractions that were analyzed by TLC and pooled into 4 subfractions (IJ-A1 to IJ-A4). The subfraction coded IJ-A2 (422.0 mg) gave a white precipitate which was identified as guaiacin $(\mathbf{1}, 136.0 \mathrm{mg}){ }^{5}$ while IJ-A3 $(52.0 \mathrm{mg})$ was fractioned by preparative TLC (silica gel and hexane:EtOAc 7:3) providing verrucosin $(\mathbf{2}, 18.0 \mathrm{mg}){ }^{6}$

The hydroalcoholic residue of the hexane extract of $V$. mollissima pericarps $(2.5 \mathrm{~g})$ was fractionated by silica gel column chromatography and eluted with hexane:EtOAc of increasing polarity, resulting in the isolation of grandisin $(3,13.8 \mathrm{mg}){ }^{7,8}$ The hexane residue of the EtOH extract from the arils ( $3.84 \mathrm{~g}$ ) showed formation of crystals, which recrystallized in $\mathrm{CH}_{2} \mathrm{Cl}_{2}$ afforded hydroxy-oxo-otobain $(4,11.0 \mathrm{mg}){ }^{9,10}$ The precipitate $(555.0 \mathrm{mg})$ of the hexane extract $V$. mollissima arils with hot $\mathrm{MeOH}$ was submitted to column chromatography on silica gel and eluted with hexane:EtOAc of increasing polarity, yielding 67 fractions which were pooled into four groups (VM-A1 to VMA4) after TLC analysis. The purification of VM-A4 by preparative TLC yielded seco-otobain $(\mathbf{5}, 12.0 \mathrm{mg}){ }^{9}$

The $\mathrm{CHCl}_{3}$ residue of the $\mathrm{EtOH}$ extract from $V$. pavonis arils $(2.4 \mathrm{~g})$ was first fractionated by column chromatography with silica gel and eluted with hexane:EtOAc of increasing polarity. This procedure yielded 17 fractions that were reduced to 5 fractions according their TLC profiles (VPA1 to VP-A5). Fraction VP-A1 (479.0 mg) was submitted to preparative TLC, and eluted with $98: 2, \mathrm{CHCl}_{3}$ :EtOAc to afford carinatin $(\mathbf{6}, 133.0 \mathrm{mg})$, carinatidin $(\mathbf{7}, 48.0 \mathrm{mg})$, dihydrocarinatin $(\mathbf{8}, 103.0 \mathrm{mg})$ and dihydrocarinatidin $(\mathbf{9}$, $60.0 \mathrm{mg}){ }^{11}$ Fraction VP-A2 $(159.0 \mathrm{mg})$, through preparative TLC (silica gel and 4:1 hexane:EtOAc), led to the isolation of dehydrodieugenol (10, $24.0 \mathrm{mg}){ }^{12}$ After several steps to column chromatography and preparative TLC, fraction VP-A3 $(197.0 \mathrm{mg})$ gave three tetrahydrofuran neolignans: galgravin $(\mathbf{1 1}, 16.0 \mathrm{mg}),{ }^{13}$ nectandrin $\mathrm{A}(\mathbf{1 2}, 7.5 \mathrm{mg})^{14}$ and galbelgin $(\mathbf{1 3}, 12.0 \mathrm{mg}){ }^{15}$ Fraction VP-A4 $(43.0 \mathrm{mg})$ was submitted to preparative TLC using 85:15 hexane:EtOAc provided the erythro $(\mathbf{1 4}, 5.4 \mathrm{mg})$ and the threo $(\mathbf{1 5}$, 
$3.0 \mathrm{mg}$ ) isomers of an oxyneolignan. ${ }^{16} \mathrm{VP}-\mathrm{A} 5(54.0 \mathrm{mg})$ provided verrucosin $(2,3.3 \mathrm{mg})$ after fractionation by column chromatography over silica gel and elution with hexane:EtOAc of increasing polarity. The $\mathrm{CHCl}_{3}$ residue of the EtOH extract from $V$. pavonis pericarps fractionated by column chromatography and eluted with hexane:EtOAc at increasing polarity provided the fractions VP-P1 to VP-P4. The purification of VP-P2 and VP-P3 by preparative TLC with hexane: $\mathrm{Me}_{2} \mathrm{CO} 4: 1$ as eluent provided carinatone (16, $4.5 \mathrm{mg})^{17}$ and nectandrin B $(\mathbf{1 7}, 3.0 \mathrm{mg}),{ }^{6}$ respectively.

The ground leaves of V. michelii $(730.0 \mathrm{~g})$ were extracted for six hours in a Soxhlet apparatus, first with hexane and then with EtOH. After that, the solutions were concentrated under reduced pressure yielding $28.4 \mathrm{~g}$ of hexane extract and $89.5 \mathrm{~g}$ of EtOH extract. Then, the crude EtOH extract was dissolved in $\mathrm{MeOH}$ :water 9:1, and partitioned with hexane and EtOAc yielding three residues. After TLC analysis, the EtOAc residue $(29.2 \mathrm{~g})$ was submitted to column chromatography using silica gel and $\mathrm{CHCl}_{3}: \mathrm{MeOH}$ in mixtures of increasing polarity, collecting 13 fractions. Fractions 1-5, after fractionation on preparative TLC using for elution the systems: hexane:EtOAc (98:2), hexane: $\mathrm{Et}_{2} \mathrm{O}$ (8:2) and $\mathrm{CHCl}_{3}: \mathrm{Me}_{2} \mathrm{CO}$ (9:1), allowed the isolation of eudesmin $(\mathbf{1 8}, 27.3 \mathrm{mg})^{18}$ and phylligenol $(\mathbf{1 9}, 4.8 \mathrm{mg}){ }^{19}$

\section{Biological assay}

\section{Antioxidant assay}

The assay was performed with DPPH (Sigma-Aldrich) in $\mathrm{MeOH}$ solution and the $50 \%$ effective dose $\left(\mathrm{ED}_{50}\right)$ was determined following the procedure described by Chang et al. ${ }^{20}$

\section{In vitro antileishmanial assay}

Promastigote forms of Leishmania amazonensis (WHO/BR/00/LT0016), L. braziliensis (MHOM/BR/75/ M2903) and L. chagasi (MHOM/BR/72/strain 46) were maintained in M199 liquid media supplemented with $10 \%$ fetal calf serum ${ }^{21}$ and $2 \%$ human urine ${ }^{22}$ at $26^{\circ} \mathrm{C}$. About $1 \times 10^{7}$ promastigote cells were separately incubated in $24-$ well plates at $26^{\circ} \mathrm{C}$ for $72 \mathrm{~h}$ in the presence of increasing concentrations of samples. The number of cells in each culture was estimated by counting in a Neubauer chamber.

\section{Results and Discussion}

The use of adsorption chromatographic methods allowed the isolation and identification of two lignoids from the fruits of Iryanthera juruensis, three from Virola mollissima, twelve from $V$. pavonis and two from $V$. michelii leaves. The identification of isolated compounds was based especially on interpretations of their ${ }^{1} \mathrm{H}$ and ${ }^{13} \mathrm{C}$ NMR spectra and compared with reported data, since these are known compounds.

As described in the literature, ${ }^{23}$ species belonging to the genus Virola accumulate lignoids with a great variety of carbon skeleta. In I. juruensis Warburg aryltetraline neolignan (1) and a tetrahydrofuran lignan (2) were found. V. mollissima fruits presented the occurrence of the aryltetralone neolignan $\mathbf{4}$ and its seco derivative (5). From V. pavonis (A. DC.) A. C. Smith neolignans of benzofuran type (6 and 7), dihydrobenzofuran type ( 8 and 9), tetrahydrofuran type $(\mathbf{2}, \mathbf{1 1 - 1 3}, \mathbf{1 7})$ and biphenyl neolignan (10) were isolated, besides two diastereomeric forms of 8.O.4'-oxyneolignan (14 and 15). The V. michelii leaves afforded two furofuran lignans, 18 and $\mathbf{1 9 .}$

The NMR data of $\mathbf{5}$ and $\mathbf{1 9}$ allowed the unambiguous assignment of chemical shifts. The structure of secolignoid 5 was suggested ${ }^{9}$ to be produced by a retro Friedel-Crafts reaction from aryltetralone neolignan. In this work, the extensive studies using NMR techniques such as gCOSY, gHSQC and gHMBC allowed the correct assignment agreeing with those previously described (see Table 1). Through a 1D nOe (Figure 2) experiment with 5 it is possible to observe the effect of the oxy-methylene hydrogens $\mathrm{H}-1 \mathrm{a}$ and $\mathrm{H}-1 \mathrm{~b}$ with the methyl hydrogens $\mathrm{CH}_{3}-3$ and of $\mathrm{H}-4$ with $\mathrm{CH}_{3}-2$. Figure 2 represents a unique possible conformation of $\mathbf{5}$, according to the observed nOe, confirming the stereochemistry previously proposed.

The literature does not register the chemical shifts of the aromatic hydrogens phylligenol $\mathbf{1 9}$, because aromatic hydrogens in the ${ }^{1} \mathrm{H}$ NMR spectrum of guaiacyl and veratryl rings present continuous absorption. An experiment was performed at $40{ }^{\circ} \mathrm{C}$, using Gaussian transformation on the FID in order to result in better resolution of aromatic hydrogens, where it was possible to observe the aromatic hydrogens (Figure 3). Between $\delta$ 6.83-6.94 can be observed hydrogens at $\delta 6.94(d, J 1.5 \mathrm{~Hz}, \mathrm{H}-2)$, at $\delta 6.91(d, J 2.0 \mathrm{~Hz}$, $\left.\mathrm{H}-2^{\prime}\right)$, at $\delta 6.89\left(d, J 8.0 \mathrm{~Hz}, \mathrm{H}-5^{\prime}\right)$, centered at $\delta 6.86(\mathrm{H}-6$ and $\mathrm{H}-5$, close to an $\mathrm{A}_{2}$ system $), \delta 6.85(d, J 8.0 \mathrm{~Hz}, \mathrm{H}-5)$, $\delta 6.85\left(d d, J 8.0\right.$ and $\left.2.0 \mathrm{~Hz}, \mathrm{H}-6^{\prime}\right)$.

The allocation of the hydrogens from ring furofuran of $\mathbf{1 6}$ was confirmed by analysis of its gCOSY spectrum, in which observed correlations are consistent with a spin system of H-7:H-8:H-9a:H-9b, H-7':H-8':H-9'a:H-9'b, besides the correlation between the hydrogens $\mathrm{H}-8: \mathrm{H}-8$ '. The analysis of this system was based by comparison of chemical shifts of hydrogens H-7 and H-7' with models available in the literature.

In our previous work, we communicated that compound 4 showed promising results in the bioautographic test and in a solid medium quantitative activities against xylophagus 
<smiles>COc1cc([C@@H]2C[C@H](C)Cc3cc(OC)c(O)cc32)ccc1O</smiles>

1<smiles>C[C@H]1[C@@H](C)C(=O)c2ccc3c(c2[C@@]1(O)c1ccc2c(c1)OCO2)OCO3</smiles>

4<smiles>[R]c1ccc([C@@H]2Oc3c(OC)cc(CC=C)cc3[C@@H]2C)cc1OC</smiles>

$$
\begin{aligned}
& \mathrm{R}=\mathrm{OMe}(\mathbf{8}) \\
& \mathrm{R}=\mathrm{OH}(\mathbf{9})
\end{aligned}
$$<smiles>COc1ccc([C@H]2O[C@@H](c3ccc(OC)c(OC)c3)[C@H](C)[C@@H]2C)cc1OC</smiles>

13<smiles>COc1cc([C]2O[C@@H](c3ccc(O)c(OC)c3)[C@H](C)[C@H]2C)ccc1O</smiles>

2<smiles>COc1ccc(C(c2ccc3c(c2)OCO3)C(C)[C@@H](C)CO)cc1OC</smiles>

5<smiles>C=CCc1cc(OC)c(O)c(-c2cc([CH+]C=O)cc(OC)c2O)c1</smiles>

10<smiles>COc1cc([C@H]2O[C@H](c3cc(OC)c(OC)c(OC)c3)[C@H](C)[C@@H]2C)cc(OC)c1OC</smiles>

3<smiles>[R]c1ccc(-c2oc3c(OC)cc(CC=C)cc3c2C)cc1OC</smiles>

$\mathrm{R}=\mathrm{OMe}$ ( 6)

$\mathrm{R}=\mathrm{OH}$ (7)<smiles>[R]c1ccc([C@H]2O[C@@H](c3ccc([R])c(OC)c3)[C@H](C)[C@@H]2C)cc1OC</smiles>

$\mathrm{R}=\mathrm{OMe}, \mathrm{R}^{\prime}=\mathrm{OMe}(\mathbf{1 1})$

$\mathrm{R}=\mathrm{OMe}, \mathrm{R}^{\prime}=\mathrm{OH}$ ( 12)<smiles>C=CCc1ccc(O[C@H](C)[C@H](O)c2ccc(OC)c(OC)c2)c(OC)c1</smiles>

erythro (14)

threo (15)<smiles>C=CCc1cc(OC)c(O)c(C(C)C(=O)c2ccc(OC)c(OC)c2)c1</smiles>

16<smiles>COc1cc([C@H]2O[C@H](c3ccc(O)c(OC)c3)[C@H](C)[C@@H]2C)ccc1O</smiles>

17<smiles>COc1ccc([C@@H]2OC[C@@H]3[C@@H](CO[C@H]3c3ccc(OC)c(OC)c3)[C@H]2c2ccc(OC)c(OC)c2)cc1</smiles>

18<smiles>COc1cc([C@@H]2OC[C@@H]3[C@@H](c4ccc(OC)c(OC)c4)OC[C@H]23)ccc1O</smiles>

19

Figure 1. Structures of lignoids. 
Table 1. ${ }^{1} \mathrm{H}$ NMR data $(11.7 \mathrm{~T}, \delta, J$ in $\mathrm{Hz}$ ) and correlations in 2D diagrams of $\mathbf{5}$

\begin{tabular}{|c|c|c|c|c|}
\hline Hydrogens & $\delta /$ ppm, multiplicity & $\begin{array}{l}\operatorname{gCOSY} \\
{ }^{1} \mathrm{H}(\delta)\end{array}$ & $\begin{array}{l}\text { gHSBC } \\
{ }^{13} \mathrm{C}(\delta)\end{array}$ & $\begin{array}{l}\mathrm{gHMBC} \\
{ }^{13} \mathrm{C}(\delta)\end{array}$ \\
\hline 1 & $3.47(d d, J 10.4 ; 6.5 \mathrm{~Hz})$ & & & \\
\hline $3.51(d d, J 10.4 ; 8.2 \mathrm{~Hz})$ & & 67.2 & 9.8 & \\
\hline 2 & $\begin{array}{l}1.77(d d d q, J 8.2 ; 6.5 ; \\
\text { unresolved; } 6.9 \mathrm{~Hz})\end{array}$ & $3.47 / 3.51$ & 36.3 & \\
\hline 3 & $\begin{array}{l}2.60((d d q, J 11.9 ; \text { unresolved; } \\
6.9 \mathrm{~Hz})\end{array}$ & 3.53 & 36.1 & \\
\hline 4 & $3.53(d, J 11.9 \mathrm{~Hz})$ & & 56.2 & $12.0 / 108.2 / 121.1$ \\
\hline \multicolumn{5}{|l|}{ /111.5/119.7 } \\
\hline $2^{\prime}$ & $6.81(d, J 2.1 \mathrm{~Hz})$ & & 111.5 & $149.2 / 137.2$ \\
\hline $5^{\prime}$ & $6.79(d, J 8.2 \mathrm{~Hz})$ & & 111.7 & \\
\hline $6^{\prime}$ & $6.86(d d, J 8.2 ; 2.1 \mathrm{~Hz})$ & 6.79 & 119.8 & 67.2 \\
\hline $2^{\prime \prime}$ & $6.81(d, J 1.5 \mathrm{~Hz})$ & & 108.2 & 148.0 \\
\hline $5^{\prime \prime}$ & $6.70(d, J 7.9 \mathrm{~Hz})$ & 6.76 & 108.4 & 139.3 \\
\hline $6^{\prime \prime}$ & $6.76(d d, J 7.9 ; 1.5 \mathrm{~Hz})$ & & 121.1 & \\
\hline 2-Me & $0.76(d, J 6.9 \mathrm{~Hz})$ & 1.77 & 12.0 & \\
\hline 3-Me & $0.70(d, J 6.9 \mathrm{~Hz})$ & 2.60 & 9.8 & \\
\hline $\mathrm{MeO}$ & $3.83(s)$ & & 56.1 & \\
\hline $\mathrm{MeO}$ & $3.87(s)$ & & 56.2 & \\
\hline $3^{\prime \prime}, 4^{\prime \prime}-\mathrm{CH}_{2} \mathrm{O}_{2}$ & $5.88(d, J 1.4 \mathrm{~Hz})$ & & & \\
\hline $5.90(d, J 1.4 \mathrm{~Hz})$ & & 101.1 & & \\
\hline
\end{tabular}

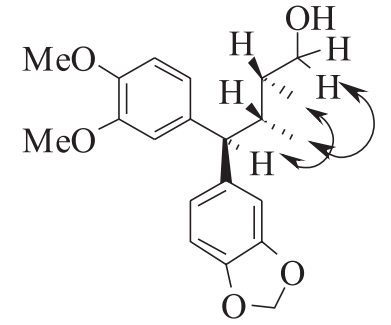

Figure 2. Observed nOe experiment of $\mathbf{5}$.

fungi Pycnoporus sanguineus, Trametes villosa and Lenzites work were determined. This experiment revealed the potential of $V$. mollissima in the development of antifungal products against wood decaying fungi. ${ }^{24}$

An antioxidant assay by the radical scavenger DPPH was performed on $V$. michelii extract, their fractions and phylligenol. The $50 \%$ effective dose $\left(\mathrm{ED}_{50}\right)$ of $16.0 \mu \mathrm{g} \mathrm{mL}^{-1}$ was found for the crude EtOH extract and for its aqueous EtOH fraction. The EtOAc fraction obtained after partition of crude extract showed a $\mathrm{ED}_{50}$ of $20.4 \mu \mathrm{g} \mathrm{mL}^{-1}$. After purification of the EtOAc fraction by preparative TLC, the major fraction lost the antioxidant activity, with $\mathrm{ED}_{50}=$ $278.0 \mu \mathrm{g} \mathrm{mL}^{-1}$. The phylligenol isolated from this fraction presented $\mathrm{ED}_{50}=108.0 \mu \mathrm{g} \mathrm{mL}^{-1}$. The $\mathrm{ED}_{50}$ determined for quercetin was $5.3 \mu \mathrm{g} \mathrm{mL}^{-1}$.

The lignoids $\mathbf{1}$ and $\mathbf{2}$ obtained from I. juruensis arils presented higher antileishmanial activity among the assayed lignoids against the promastigote forms of Leishmania amazonensis when compared with promastigote forms of $L$.
Table 2. Sensitivity of Leishmania sp. promastigote forms to lignoids

\begin{tabular}{lccc}
\hline Lignoids & L. amazonensis & L. braziliensis & L. chagasi \\
\cline { 2 - 4 } & \multicolumn{3}{c}{$\mathrm{IC}_{50} /(\mu \mathrm{g} \mathrm{mL})^{-1}$} \\
\hline $\mathbf{1}$ & 45.0 & 98.0 & 184.0 \\
$\mathbf{2}$ & 27.0 & 100.0 & 170.0 \\
$\mathbf{6}$ & $>250$ & $>250$ & $>250$ \\
$\mathbf{7}$ & 76.0 & $>250$ & $>250$ \\
$\mathbf{8}$ & 100.0 & $>250$ & $>250$ \\
$\mathbf{9}$ & $>250$ & $>250$ & $>250$ \\
$\mathbf{1 0}$ & 148.0 & 150.0 & $>250$ \\
$\mathbf{1 1}$ & $>250$ & $>250$ & $>250$ \\
$\mathbf{1 2}$ & $>250$ & $>250$ & $>250$ \\
$\mathbf{1 3}$ & $>250$ & $>250$ & $>250$ \\
$\mathbf{1 4}$ & 239.0 & 230.0 & $>250$ \\
$\mathbf{1 5}$ & $>250$ & $>250$ & $>250$ \\
$\mathbf{1 7}$ & $>250$ & $>250$ & $>250$ \\
\hline
\end{tabular}

braziliensis and L. chagasi, as shown in the Table 2. The 50\% inhibition concentration, $\mathrm{IC}_{50}$, was 27.0 and $45.0 \mu \mathrm{g} \mathrm{mL} \mathrm{m}^{-1}$ of $\mathbf{1}$ and $\mathbf{2}$, observed for $L$. amazonensis, suggests further studies for potential use of these compounds.

\section{Spectrometric data}

Rel-(8R,7'S,8'S)-4,4'-dihydroxy-3,3'-dimethoxy- $\Delta^{1,3,5, l^{\prime}, 3^{\prime}, 5^{\prime} \text { - }}$ 8.8',6.7'-neolignan (1), guaiacin

${ }^{1} \mathrm{H}$ NMR $\left(200 \mathrm{MHz}, \mathrm{CDCl}_{3}\right), \delta: 6.54(1 \mathrm{H}, s, \mathrm{H}-6) ; 6.26$ $(1 \mathrm{H}, s, \mathrm{H}-3) ; 2.59(1 \mathrm{H}, d d, J 15.8 ; 10.1 \mathrm{~Hz}, \mathrm{H}-7 \mathrm{ax}) ; 2.75$ 


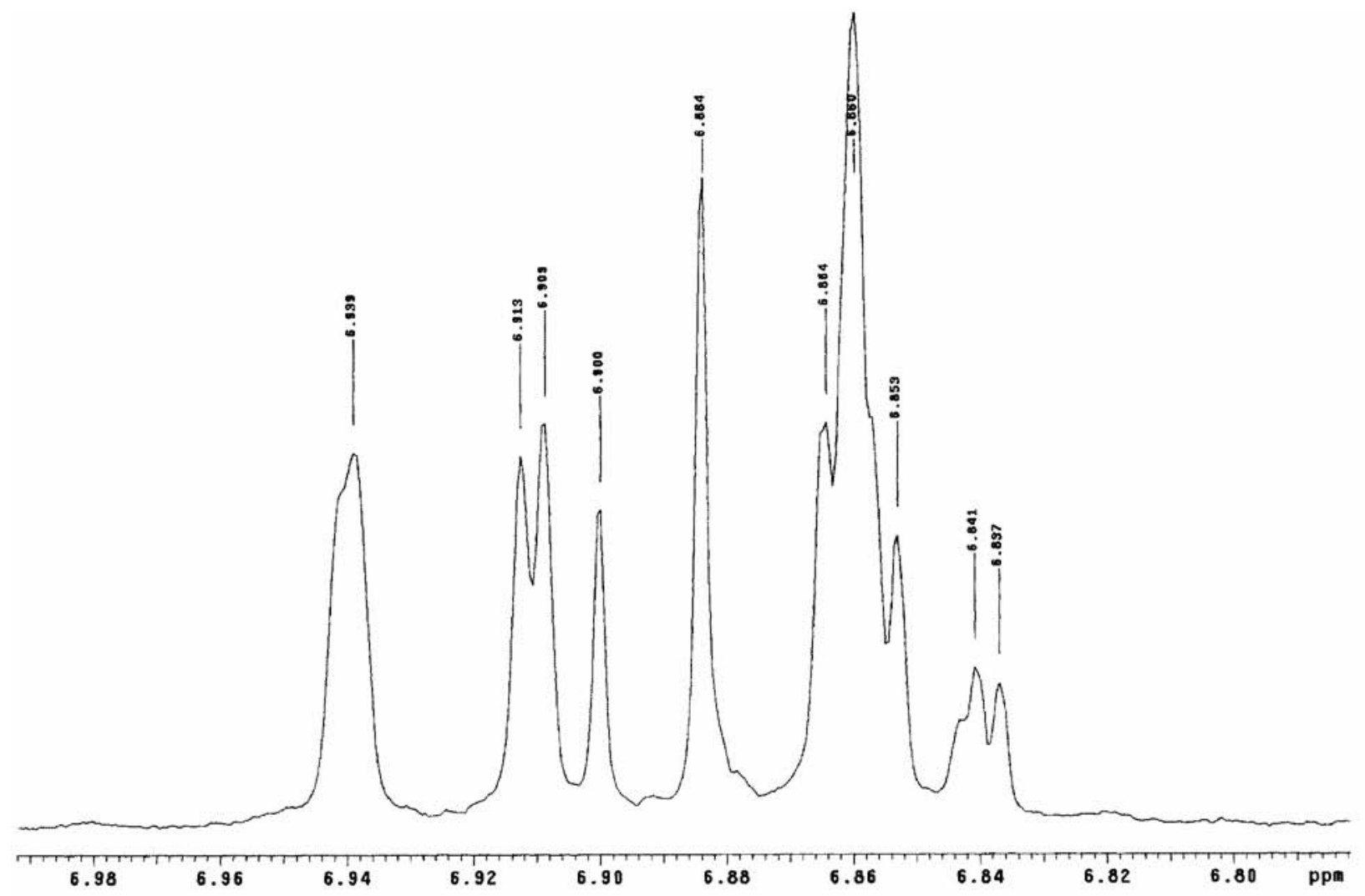

Figure 3. Aromatic region in ${ }^{1} \mathrm{H}$ NMR spectrum of $19\left(500 \mathrm{MHz}, \mathrm{CD}_{3} \mathrm{CN}\right)$.

$(1 \mathrm{H}, d d, J 15.8 ; 4.4 \mathrm{~Hz}, \mathrm{H}-7 \mathrm{eq}) ; 1.52\left(1 \mathrm{H} / 1 \mathrm{H}, m, \mathrm{H}-8 / 8^{\prime}\right)$; 1.07 (3H, $d, J 6.2 \mathrm{~Hz}, \mathrm{H}-9) ; 6.55\left(1 \mathrm{H}, d, J 2.2 \mathrm{~Hz}, \mathrm{H}-2^{\prime}\right)$; $6.83\left(1 \mathrm{H}, d, J 8.4 \mathrm{~Hz}, \mathrm{H}-5{ }^{\prime}\right) ; 6.63(1 \mathrm{H}, d d, J 8.4 ; 2.2 \mathrm{~Hz}$, H-6'); $3.37(1 \mathrm{H}, d, J 10.3 \mathrm{~Hz}, \mathrm{H}-7$ '); $0.85(3 \mathrm{H}, d, J 6.2 \mathrm{~Hz}$, H-9'); 3.82 (3H, $s, \mathrm{MeO}-3$ ); 3.84 (3H, $s, \mathrm{MeO}-3^{\prime}$ ); 5.28 $(1 \mathrm{H}, s, \mathrm{OH}-\mathrm{C} 4) ; 5.48(1 \mathrm{H}, s, \mathrm{OH}-\mathrm{C} 4$ ').

${ }^{13} \mathrm{C}$ NMR $\left(50 \mathrm{MHz}, \mathrm{CDCl}_{3}\right): \delta: 128.4(\mathrm{C}-1) ; 133.5(\mathrm{C}-$ 2); 115.5 (C-3); 143.3 (C-4); 143.9 (C-5); 110.0 (C-6); 39.1 (C-7); 43.7 (C-8); 20.0 (C-9); 138.3 (C-1'); 111.5 (C-2'); 146.5 (C-3'); 144.6 (C-4'); 113.9 (C-5'); 122.5 (C-6'); 54.2 (C-7'); 35.6 (C-8'); 17.2 (C-9'); 55.8 (MeO-3); 55.9 (MeO-3').

MS (70 eV), m/z (Rel. Int. \%) M+ 328 (100), 241 (94), 271 (43), 189 (32), 136 (143).

Rel-(7S, 8S, 7'R, 8'S)-4,4'-dihydroxy-3,3'-dimethoxy$\Delta^{1,3,5,1^{\prime}, 3,5^{\prime}-8.8}$ ',7.O.7'-neolignan (2), verrucosin

${ }^{1} \mathrm{H}$ NMR $\left(200 \mathrm{MHz}, \mathrm{CDCl}_{3}\right), \delta: 5.11(1 \mathrm{H}, d, J 8.5 \mathrm{~Hz}$, H-7); $4.40(1 \mathrm{H}, d, J 9.0 \mathrm{~Hz}, \mathrm{H}-7$ ' $) ; 2.26(1 \mathrm{H}, m, \mathrm{H}-8) ; 1.76$ $(1 \mathrm{H}, m, \mathrm{H}-8$ '); $1.06(3 \mathrm{H}, d, J 6.5 \mathrm{~Hz}, \mathrm{H}-9) ; 0.66(3 \mathrm{H}, d, J 7.0$ Hz, H-9'); 7.05-6.80 (6H, $m$, Ar-H); 3.91 (3H, $s, \mathrm{MeO}-3$ ); 3.85 (3H, s, MeO-3'); 5.63 (1H, sl, OH-4/4').

${ }^{13} \mathrm{C}$ NMR $\left(50 \mathrm{MHz}, \mathrm{CDCl}_{3}\right), \delta: 132.8(\mathrm{C}-1) ; 109.5(\mathrm{C}-$ 2); 146.5 (C-3); 145.2 (C-4); 114.1 (C-5); 119.3 (C-6); 87.3 (C-7); 47.8 (C-8); 14.9 (C-9); 132.2 (C-1'); 109.8 (C-2');
146.2 (C-3'); 144.6 (C-4'); 113.8 (C-5'); 119.9 (C-6'); 83.1 (C-7'); 46.0 (C-8'); 15.0 (C-9'); 55.8 (MeO-3/3').

MS (70 eV), m/z (Rel. Int. \%): M+ 192 (100), 151 (64), 177 (57), 152 (21), 164 (18).

Rel-(7R,8S,7'R, 8'R)-3,4,5,3',4',5'-hexamethoxy- $\Delta^{1,3,5,1^{\prime}, 3^{\prime}, 5^{\prime} \text { - }}$ 8.8',7.O.7'-neolignan (3), grandisin

${ }^{1} \mathrm{H}$ NMR (300 MHz, $\mathrm{CDCl}_{3}$ ), $\delta: 6.45(2 \mathrm{H}, l s, \mathrm{H}-2 / 6)$; $4.27(1 \mathrm{H}, d, J 9.0 \mathrm{~Hz}, \mathrm{H}-7) ; 1.80(1 \mathrm{H}, m, \mathrm{H}-8) ; 1.12$ (3H, d, J $6.5 \mathrm{~Hz}, \mathrm{H}-9) ; 6.58$ (2H, s, H-2'/6'); 5.12 (1H, d, J 8.5 Hz, H-7'); 2.25 (1H, m, H-8'); 0.69 (3H, d, J 7.0 Hz, H-9'); 3.81 (3H, s, MeO-4); 3.83 (3H, s, MeO-4'); 3.86 (3H, s, MeO-3); 3.87 (3H, s, MeO-3'); 3.88 (3H, s, MeO-5); 3.89 (3H, s, MeO-5').

${ }^{13} \mathrm{C}$ NMR (75 MHz, $\left.\mathrm{CDCl}_{3}\right), \delta: 134.5$ (C-1); 134.1 (C-1'); 103.8 (C-2/2'/6); 153.5 (C-3); 153.3 (C-3'); 136.9 (C-4); 136.7 (C-4'); 153.5 (C-5); 153.1 (C-5'); 104.1 (C6'); 87.6 (C-7); 87.4 (C-7'); 46.3 (C-8); 48.1 (C-8'); 15.1 (C-9); 14.3 (C-9'); $56.3\left(\mathrm{MeO}-3 / 5 / 3^{\prime} / 5^{\prime}\right)$; 61.1 (MeO-4/4').

Rel-(8S,7'S,8'R)-7'-hydroxy-3,4,3',4'-dimethylenedioxy-

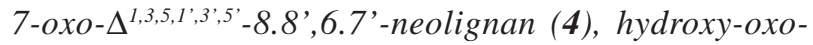
otobain

${ }^{1} \mathrm{H}$ NMR (300 MHz, $\mathrm{CDCl}_{3}$ ), $\delta: 1.20(3 \mathrm{H}, d, J 7.0 \mathrm{~Hz}$, H-9); 0.93 (3H, $\left.d, J 7.0 \mathrm{~Hz}, \mathrm{H}-9^{\prime}\right) ; 2.90$ (1H, $d q, J$ 7.0; 
unresolved $\mathrm{Hz}, \mathrm{H}-8) ; 2.14$ (1H, dq, $J$ 7.0; unresolved $\mathrm{Hz}$, H-8'); $7.71(1 \mathrm{H}, d, J 8.0 \mathrm{~Hz}, \mathrm{H}-6) ; 6.79$ (1H, $d d, J$ 8.5; 1.2 $\left.\mathrm{Hz}, \mathrm{H}-6^{\prime}\right) ; 6.89$ (1H, $\left.d, J 8.0 \mathrm{~Hz}, \mathrm{H}-5\right)$; $6.74(1 \mathrm{H}, d, J 8.5$ $\left.\mathrm{Hz}, \mathrm{H}-5^{\prime}\right) ; 6.81\left(1 \mathrm{H}, d, J 1.2 \mathrm{~Hz}, \mathrm{H}-2^{\prime}\right) ; 5.72$ and $5.86(2 \mathrm{H}$, d, J $\left.1.2 \mathrm{~Hz}, \mathrm{OCH}_{2} \mathrm{O}\right) ; 5.97\left(2 \mathrm{H}, \mathrm{s}, \mathrm{OCH}_{2} \mathrm{O}\right)$.

${ }^{13} \mathrm{C} \mathrm{NMR}\left(75 \mathrm{MHz}, \mathrm{CDCl}_{3}\right), \delta: 126.6(\mathrm{C}-1) ; 140.4\left(\mathrm{C}-1^{\prime}\right)$; 128.4 (C-2); 106.6 (C-2'); 144.5 (C-3); 146.2 (C-3'); 152.5 (C-4); 147.3 (C-4'); 108.8 (C-5); 107.4 (C-5'); 122.8 (C6); 118.9 (C-6'); 198.5 (C-7); 74.8 (C-7'); 43.3 (C-8); 46.7 (C-8'); 121.0 (C-9); 12.0 (C-9'); 100.9 and $101.0\left(\mathrm{OCH}_{2} \mathrm{O}\right)$.

Rel-(2S,3R,4R)-2,3-dimethyl-4-(3',4'-dimethoxyphenyl)4-(3",4"'-methylenedioxyphenyl)-butan-1-ol (5), secootobain

${ }^{1} \mathrm{H}$ NMR (300 MHz, $\mathrm{CDCl}_{3}$ ), see the Table $1 .{ }^{13} \mathrm{C}$ NMR (75 MHz, $\mathrm{CDCl}_{3}$ ), $\delta$ : 67.2 (C-1); 36.3 (C- 2); 36.1 (C-3); 56.2 (C-4); 12.0 ( $\left.\mathrm{H}_{3} \mathrm{C}-2\right)$; 9.8 ( $\left.\mathrm{H}_{3} \mathrm{C}-3\right)$; 137.2 (C-1'); 108.2 (C-2'); 149.2 (C-3'); 147.6 (C-4'); 111.5 (C-5'); 119.8 (C-6’); 139.3 (C-1”); 108.4 (C-2”); 148.0 (C-3”); 145.9 (C-4”); 111.7 (C-5”); 121.1 (C-6”); $101.1\left(\mathrm{OCH}_{2} \mathrm{O}\right) ; 56.2$ (MeO-3); 56.1 (MeO-4).

3,4,3'-Trimethoxy- $\Delta^{1,3,5,7,1^{\prime}, 3^{\prime}, 5^{\prime}, 8^{\prime}}$-8. ', ', $7.0 .4^{\prime}$ 'neolignan (6), carinatin

${ }^{1} \mathrm{H}$ NMR (200 MHz, $\left.\mathrm{CDCl}_{3}\right), \delta: 7.37-7.33(2 \mathrm{H}, m$, $\mathrm{H}-2 / 6) ; 6.96$ (1H, $d, J 9.0 \mathrm{~Hz}, \mathrm{H}-5) ; 2.43$ (3H, s, H-9); 6.65 (1H, ls, H-2'); 6.94 (1H, d, J $1.2 \mathrm{~Hz}, \mathrm{H}-6$ '); 3.49 (2H, d, J 7.0 Hz, H-7'); 6.06 (1H, ddt, J 16.5; 10.0; 6.5 Hz, H-8'); 5.19-5.09 (2H, m, H-9'); 3.99 (3H, s, MeO-3); 3.94 (3H, s, MeO-4); 4.03 (3H, s, MeO-3').

${ }^{13} \mathrm{C}$ NMR (50 MHz, $\mathrm{CDCl}_{3}$ ), $\delta: 133.0(\mathrm{C}-1) ; 107.5$ (C2); 148.9 (C-3 and C-4); 110.9 (C-5); 119.9 (C-6); 151.2 (C-7); 110.3 (C-8); 9.6 (C-9); 135.1 (C-1'); 110.0 (C-2'); 144.7 (C-3’); 141.5 (C-4'); 124.3 (C-5'); 111.1 (C-6'); 40.6 (C-7’); 138.0 (C-8'); 115.6 (C-9'); 56.1 (MeO-3); 56.0 (MeO-4); 55.9 (MeO-3’).

4-Hydroxy-3,3'-dimethoxy- $\Delta^{1,3,5,7,1^{\prime}, 3^{\prime}, 5^{\prime}, 8^{\prime}-8.5^{\prime}, 7.0 .4^{\prime} \text { - }}$ neolignan (7), carinatidin

${ }^{1} \mathrm{H}$ NMR (200 MHz, $\mathrm{CDCl}_{3}$ ), $\delta: 7.33-7.25(2 \mathrm{H}, m$, $\mathrm{H}-2 / 6) ; 6.99$ (1H, d, J 7.9 Hz, H-5); 2.41 (3H, s, H-9); 6.64 (1H, ls, H-2'); 6.93 (1H, ls, H-6'); 3.48 (2H, d, J $6.5 \mathrm{~Hz}$, H-7'); 6.02 (1H, ddt, J 16.5; 10.0; 6.5 Hz, H-8'); 5.18-5.07 (2H, m, H-9'); 3.98 (3H, s, MeO-3); 5.77 (1H, ls, OH-4); 4.02 (3H, s, MeO-3').

${ }^{13} \mathrm{C}$ NMR (50 MHz, $\mathrm{CDCl}_{3}$ ), $\delta: 133.0$ (C-1); 107.5 (C- 2); 146.6 (C-3); 145.7 (C-4); 114.4 (C-5); 120.6 (C-6); 151.4 (C-7); 110.0 (C-8); 9.6 (C-9); 135.1 (C-1'); 109.5 (C2'); 144.7 (C-3'); 141.4 (C-4'); 123.8 (C-5'); 111.0 (C-6'); 40.6 (C-7’); 138.0 (C-8’); 115.6 (C-9'); 56.1 (MeO-3/3’).
Rel-(7S,8S)-3,4,3'-trimethoxy- $\Delta^{1,3,5,1^{\prime}, 3^{\prime}, 5^{\prime}, 8^{\prime}}$-8.5', 7.O.4'neolignan (8), dihydrocarinatin

${ }^{1} \mathrm{H}$ NMR (200 MHz, $\left.\mathrm{CDCl}_{3}\right), \delta: 7.00-6.95(2 \mathrm{H}, m$, $\mathrm{H}-2 / 6) ; 6.85(1 \mathrm{H}, d, J 8.0 \mathrm{~Hz}, \mathrm{H}-5) ; 5.11(1 \mathrm{H}, d, J 9.5 \mathrm{~Hz}$, H-7); 3.51-3.43 (1H, $m, \mathrm{H}-8)$; 1.38 (3H, d, J $6.5 \mathrm{~Hz}, \mathrm{H}-9$ ); 6.62 (1H, s, H-2'); 6.64 (1H, s, H-6'); 3.37 (2H, $d, J 6.5 \mathrm{~Hz}$, H-7'); 5.99 (1H, ddt, J 17.0; 10.0; 6.5 Hz, H-8'); 5.16-5.06 (2H, $m, \mathrm{H}-9$ '); 3.89 (9H, s, MeO-3/4/3').

${ }^{13} \mathrm{C} \mathrm{NMR}\left(\mathrm{CDCl}_{3}, 50 \mathrm{MHz}\right), \delta: 137.9$ (C-1); 109.5 (C2); 149.1 (C-3 and C-4); 110.7 (C-5); 119.3 (C-6); 93.6 (C-7); 45.7 (C-8); 17.5 (C-9); 133.1 (C-1'); 111.8 (C-2’); 145.1 (C-3’); 144.1 (C-4'); 133.5 (C-5’); 115.6 (C-6'); 40.2 (C-7’); 137.9 (C-8'); 115.6 (C-9'); 55.9 (MeO-3/4/3').

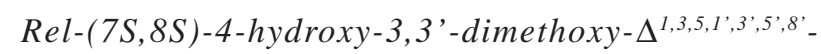
8.5',7.O.4'-neolignan (9), dihydrocarinatidin

${ }^{1} \mathrm{H}$ NMR (200 MHz, $\mathrm{CDCl}_{3}$ ), $\delta: 6.99-6.91(3 \mathrm{H}, m$, $\mathrm{H}-2 / 5 / 6) ; 5.09$ (1H, d, J $10.0 \mathrm{~Hz}, \mathrm{H}-7) ; 3.50-3.45(1 \mathrm{H}, m$, H-8); 1.38 (3H, d, J 7.0 Hz, H-9); 6.62 (1H, s, H-2'); 6.64 (1H, s, H-6'); 3.37 (2H, d, J 6.5 Hz, H-7'); 5.98 (1H, ddt, J 17.0; 10.0; 6.5 Hz, H-8'); 5.17-5.06 (2H, m, H-9'); 3.89 (6H, s, MeO-3/3'); 5.31 (1H, s, OH-4).

${ }^{13} \mathrm{C}$ NMR (50 MHz, $\mathrm{CDCl}_{3}$ ), $\delta: 132.2$ (C-1); 108.9 (C2); 146.7 (C-3); 145.7 (C-4); 114.0 (C-5); 119.9 (C-6); 93.7 (C-7); 45.8 (C-8); 17.4 (C-9); 133.5 (C-1'); 111.8 (C-2'); 144.0 (C-3'); 108.9 (C-4'); 133.2 (C-5'); 115.6 (C-6'); 40.2 (C-7’); 137.9 (C-8'); 55.9 (MeO-3/3’).

4,4'-Dihydroxy-3,3'-dimethoxy- $\Delta^{1,3,5,8,1^{\prime}, 3^{\prime}, 5^{\prime}, 8^{\prime}}$-5.5'neolignan (10), dehydrodieugenol

${ }^{1} \mathrm{H}$ NMR (200 MHz, $\left.\mathrm{CDCl}_{3}\right), \delta: 6.74(2 \mathrm{H}, d, J 2.0 \mathrm{~Hz}$, $\left.\mathrm{H}-2 / 2^{\prime}\right) ; 6.76$ (2H, d, J $\left.2.0 \mathrm{~Hz}, \mathrm{H}-6 / 6^{\prime}\right) ; 3.37$ (4H, $d, J 6.5$ Hz, H-7/7'); 5.97 (2H, ddt, J 17.0; 10.0; 6.5 Hz, H-8/8'); 5.17-5.05 (4H, m, H-9/9'); 3.92 (6H, s, MeO-5/5').

${ }^{13} \mathrm{C}$ NMR (50 MHz, $\left.\mathrm{CDCl}_{3}\right), \delta: 131.9$ (C-1/1'); 123.1 (C-2/2'); 124.4 (C-3/3'); 140.8 (C-4/4'); 147.2 (C-5/5'); 110.6 (C-6/6'); 39.9 (C-7/7'); 137.6 (C-8/8'); 115.7 (C9/9'); 56.0 (MeO-5/5').

Rel-(7S,8S,7'R, 8'R)-3,4,3 ',4'-tetramethoxy- $\Delta^{1,3,5,1^{\prime}, 3^{\prime}, 5^{\prime} \text { - }}$ 8.8',7.O.7'-neolignan (11), galgravin

${ }^{1} \mathrm{H}$ NMR (200 MHz, $\left.\mathrm{CDCl}_{3}\right), \delta: 4.53(2 \mathrm{H}, d, J 6.5$ Hz, H-7/7'); 2.34 (2H, m, H-8/8'); 1.05 (6H, $d, J 6.5$ Hz, H-9/9'); 6.99-6.85 (6H, m, Ar-H); 3.89 (6H, s, MeO-3/3'); 3.88 (6H, s, MeO-4/4').

${ }^{13} \mathrm{C}$ NMR (50 MHz, $\mathrm{CDCl}_{3}$ ), $\delta: 134.7$ (C-1/1'); 109.6 (C-2/2'); 148.9 (C-3/3'); 148.4 (C-4/4'); 110.9 (C-5/5'); 118.5 (C-6/6'); 87.2 (C-7/7'); 55.8 (MeO-3/4/3'/4'); 44.3 (C-8/8'); 12.9 (C-9/9').

MS (70 eV), m/z (Rel. Int. \%): M+ 206 (100), 191 (61), 372 (24), 165 (20); 178 (13); 194 (4), 166 (4). 
Rel-(7S,8S,7'R, 8'R)-4'-hydroxy-3,4,3'-trimethoxy$\Delta^{1,3,5,1^{\prime}, 3^{\prime}, 5^{\prime}-8.8}$ ',7.O.7'-neolignan (12), nectandrin $A$

${ }^{1} \mathrm{H}$ NMR $\left(200 \mathrm{MHz}, \mathrm{CDCl}_{3}\right), \delta: 4.67(2 \mathrm{H}, d, J 9.0$ Hz, H-7/7'); 1.79 (2H, m, H-8/8'); 1.05 (6H, d, J 6.0 Hz, H-9/9'); 7.0-6.83 (6H, m, Ar-H); 3.92 (6H, s, MeO-3/3'); 3.86 (6H, s, MeO-4/4').

${ }^{13} \mathrm{C}$ NMR (50 MHz, $\mathrm{CDCl}_{3}$ ), $\delta: 134.8$ (C-1); 109.7 (C2); 148.9 (C-3); 148.4 (C-4); 110.9 (C-5); 118.5 (C-6); 87.2 (C-7); 44.2 (C-8); 12.8 (C-9); 134.1 (C-1'); 109.1 (C-2'); 146.4 (C-3'); 145.0 (C-4'); 114.1 (C-5'); 119.2 (C-6'); 87.3 (C-7'); 44.3 (C-8'); 12.9 (C-9'); 55.9 (MeO-4); 55.8 (MeO-3/3').

MS (70 eV), m/z (Rel. Int. \%): M+ 206 (100), 191 (74), 178 (44), 372 (20), 194 (20), 165 (7).

Rel-(7S,8S,7'S, 8'S)-3,4,3', 4'-tetramethoxy- $\Delta^{1,3,5,1^{\prime}, 3^{\prime}, 5^{\prime} \text { - }}$ 8.8',7.O.7'-neolignan (13), galbelgin

${ }^{1} \mathrm{H}$ NMR (200 MHz, $\mathrm{CDCl}_{3}$ ), $\delta: 4.49(2 \mathrm{H}, d, J 6.0$ Hz, H-7/7'); 2.33 (2H, m, H-8/8'); 1.03 (6H, d, J $6.5 \mathrm{~Hz}$, H-9/9'); 7.04-6.85 (6H, m, Ar-H); 3.88 (6H, s, MeO-3/3'); 5.59 (2H, s, OH-C4/4').

${ }^{13} \mathrm{C}$ NMR (50 MHz, $\mathrm{CDCl}_{3}$ ), $\delta: 134.9$ (C-1/1'); 109.1 (C-2/2'); 149.0 (C-3/3'); 148.5 (C-4/4'); 110.8 (C-5/5'); 118.6 (C-6/6'); 88.3 (C-7/7'); 55.8 (MeO-3/4/3'/4'); 50.9 (C-8/8'); 13.8 (C-9/9').

MS (70 eV), m/z (Rel. Int. \%): $\mathrm{M}^{+} 192$ (100), 177 (54), 151 (44), 164 (17), 152 (14); 180 (10), 344 (7).

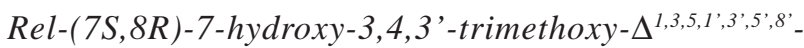
8.O.4'-neolignan (14), erythro

${ }^{1} \mathrm{H}$ NMR (200 MHz, $\left.\mathrm{CDCl}_{3}\right), \delta: 7.00-6.75$ (6H, m, Ar$\mathrm{H}) ; 4.84(1 \mathrm{H}, d, J 2.6 \mathrm{~Hz}, \mathrm{H}-7) ; 4.33(1 \mathrm{H}, m, \mathrm{H}-8) ; 1.18$ (3H, $d, J 6.6 \mathrm{~Hz}, \mathrm{H}-9)$; 3.37 (2H, $d, J$ 7.0 Hz, H-7'); 5.98 (1H, ddt, J 16.6; 10.3; 6.6 Hz, H-8'); 5.16-5.07 (2H, m, H-9'); 3.90 (3H, s, MeO-3); 3.89 (3H, s, MeO-3'); 3.88 (3H, s, MeO-4).

${ }^{13} \mathrm{C}$ NMR (50 MHz, $\mathrm{CDCl}_{3}$ ), $\delta: 132.5$ (C-1); 110.7 (C-2); 148.1 (C-3); 151.4 (C-4); 109.4 (C-5); 121.1 (C-6); 73.4 (C-7); 82.5 (C-8); 13.5 (C-9); 135.5 (C-1'); 112.4 (C-2'); 148.8 (C-3'); 144.7 (C-4'); 118.4 (C-5'); 119.9 (C-6’); 39.9 (C-7’); 137.2 (C-8'); 115.9 (C-9'); 55.8 (MeO-3/4/3’).

Rel-(7R,8R)-7-hydroxy-3,4,3'-trimethoxy- $\Delta^{1,3,5,1^{\prime}, 3^{\prime}, 5^{\prime}, 8^{\prime} \text { - }}$ 8.O.4'-neolignan (15), threo

${ }^{1} \mathrm{H}$ NMR $\left(200 \mathrm{MHz}, \mathrm{CDCl}_{3}\right), \delta: 6.97-6.72(6 \mathrm{H}, \mathrm{m}, \mathrm{Ar}-\mathrm{H})$; 4.64 (1H, $d, J 8.3 \mathrm{~Hz}, \mathrm{H}-7)$; 4.06 (1H, m, H-8); 1.17 (3H, $d$, $J$ $6.1 \mathrm{~Hz}, \mathrm{H}-9)$; 3.37 (2H, $\left.d, J 6.6 \mathrm{~Hz}, \mathrm{H}-7^{\prime}\right)$; 5.95 (1H, ddt, J 16.6; 10.5; 6.6 Hz, H-8'); 5.15-5.07 (2H, m, H-9'); 3.91 (3H, s, MeO-3); 3.89 (3H, s, MeO-3'); 3.88 (3H, s, MeO-4).

${ }^{13} \mathrm{C}$ NMR (50 MHz, $\mathrm{CDCl}_{3}$ ), $\delta: 132.3$ (C-1); 110.3 (C2); 148.2 (C-3); 150.0 (C-4); 109.6 (C-5); 120.3 (C-6);
77.5 (C-7); 82.8 (C-8); 16.2 (C-9); 134.3 (C-1'); 111.9 (C-2'); 148.4 (C-3'); 145.3 (C-4'); 118.2 (C-5'); 119.3 (C-6'); 39.3 (C-7'); 136.8 (C-8'); 115.2 (C-9'); 55.2 (MeO-3/4/3').

Rel-(8S)-4'-hydroxy-3,4,3'-trimethoxy-7-oxo- $\Delta^{1,3,5,1^{\prime}, 3,3^{\prime}, 8^{\prime},}$ 8.5'-neolignan (16), carinatone

${ }^{1} \mathrm{H}$ NMR $\left(200 \mathrm{MHz}, \mathrm{CDCl}_{3}\right), \delta: 7.62(1 \mathrm{H}, d, J 2 \mathrm{~Hz}$, $\mathrm{H}-2) ; 6.82(1 \mathrm{H}, d, J 8.5 \mathrm{~Hz}, \mathrm{H}-5) ; 7.73(1 \mathrm{H}, d d, J 8.5 ; 2$ $\mathrm{Hz}, \mathrm{H}-6) ; 5.06(1 \mathrm{H}, q, J 7 \mathrm{~Hz}, \mathrm{H}-8) ; 1.47(3 \mathrm{H}, d, J 6.5 \mathrm{~Hz}$, H-9); $6.53\left(1 \mathrm{H}, d, J 2 \mathrm{~Hz}, \mathrm{H}-2^{\prime}\right)$; 6.56 (1H, $\left.d, J 2 \mathrm{~Hz}, \mathrm{H}^{\prime} 6^{\prime}\right)$; $3.23\left(2 \mathrm{H}, \mathrm{d}, J 6.5 \mathrm{~Hz}, \mathrm{H}^{-} 7^{\prime}\right) ; 5.87$ (1H, $\left.m, \mathrm{H}-8^{\prime}\right) ; 5.03-4.97$ (2H, $\left.m, \mathrm{H}-9^{\prime}\right)$; 3.89 (6H, $\left.s, \mathrm{MeO}-3 / 5^{\prime}\right)$; 3.87 (3H, s, MeO4); $5.31(1 \mathrm{H}, s, \mathrm{OH}-4$ ').

Rel-(7S, 8S, 7'R, 8'R)-4,4'-dihydroxy-3,3'-dimethoxy$\Delta^{1,3,5,1^{\prime}, 3^{\prime}, 5^{\prime}}-8.8^{\prime}, 7.0 .7^{\prime}$-neolignan (17), nectandrin B

${ }^{1} \mathrm{H}$ NMR $\left(200 \mathrm{MHz}, \mathrm{CDCl}_{3}\right), \delta: 4.52 / 4.51(1 \mathrm{H}, d, J$ $\left.6.5 \mathrm{~Hz}, \mathrm{H}-7 / 7^{\prime}\right) ; 2.34$ (1H, $\left.m, \mathrm{H}-8 / 8^{\prime}\right) ; 1.05 / 1.04(3 \mathrm{H}, d$, $\left.J 6.5 \mathrm{~Hz}, \mathrm{H}-9 / 9^{\prime}\right) ; 6.99-6.85$ (6H, $\left.m, \mathrm{Ar}-\mathrm{H}\right) ; 3.89$ (6H, $s$, MeO-3/3'); 3.88 (3H, s, MeO-4'); 5.59 (1H, $s, \mathrm{OH}-4$ ').

${ }^{13} \mathrm{C}$ NMR (50 MHz, $\left.\mathrm{CDCl}_{3}\right), \delta: 134.2\left(\mathrm{C}-1 / 1^{\prime}\right) ; 109.1$ (C-2/2'); 146.4 (C-3/3'); 145.0 (C-4/4'); 114.1 (C-5/5'); 119.3 (C-6/6'); 87.3 (C-7/7'); 55.8 (MeO-3/3'); 44.3 (C8/8'); 12.9 (C-9/9').

MS (70 eV), m/z (Rel. Int. \%): $\mathrm{M}^{+} 192$ (100), 206 (81), 191 (66), 177 (56), 151 (47), 178 (16), 152 (5), 164 (9).

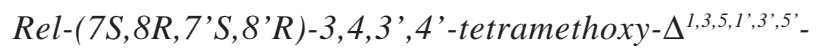
8.8',9.O.7',7.O.9'-lignan (18), eudesmin

${ }^{1} \mathrm{H}$ NMR (500 MHz, $\left.\mathrm{CDCl}_{3}\right), \delta: 6.91(2 \mathrm{H}, d, J 2.0 \mathrm{~Hz}$, H-2/2'); 6.84 (2H, d, J $8.0 \mathrm{~Hz}, \mathrm{H}-5 / 5$ '); 6.88 (2H, dd, J 8.5; $\left.2.0 \mathrm{~Hz}, \mathrm{H}-6 / 6^{\prime}\right) ; 4.76$ (2H, d, J 4.5 Hz, H-7/7'); 3.11 (2H, $d t d, J$ 9.5; 4.5; $2.0 \mathrm{~Hz}, \mathrm{H}-8 / 8$ '); 4.26 (2H, dd, J 9.0; 6.5 Hz, H-9a/9’a); 3.84-3.86 (2 H, m, H-9b/9’b); 3.88 (6H, s, MeO-4/4'); 3.90 (6H, s, MeO-3,3').

${ }^{13} \mathrm{C}$ NMR (125 MHz, $\left.\mathrm{CDCl}_{3}\right), \delta: 133.6$ (C-1/1'); 109.3 (C-2/2'); 148.7(C-3/3'); 149.3 (C-4/4'); 111.1 (C-5/5'); 118.3 (C-6/6'); 85.8 (C-7/7'); 54.2 (C-8/8'); 71.8 (C-9/9'); 55.9 (MeO-3/3'); 56.0 (MeO-4/4').

MS (70 eV), m/z (Rel. Int. \%): M $^{+} 386$ (100), 151 (31), 165 (22), 356 (13).

Rel-(7S,8R,7'R,8'R)-4-hydroxy-3,3',4'-trimethoxy$\Delta^{1,3,3,1,1^{\prime}, 3^{\prime}, 5^{\prime}-8.8^{\prime}, 9 . O .7^{\prime}, 7.0 .9 '-l i g n a n}$ (19), phylligenol

${ }^{1} \mathrm{H}$ NMR (500 MHz, $\left.\mathrm{CD}_{3} \mathrm{CN}\right), \delta: 6.99 / 6.97(2 \mathrm{H}, d$, $J$ 1.5/2.0 Hz, H-2/2'); 6.81/6.94 (2H, $d, J$ 8.0/8.0 Hz, $\mathrm{H}-5 / 5$ '); 6.87/6.94 $(1 \mathrm{H} / 1 \mathrm{H}, d d, J$ 8.0; $1.5 / 8.0 ; 2.0 \mathrm{~Hz}$, H-6/6'); 4.41/4.86 (1H/1H, d, J 7.0/5.5 Hz, H-7/7'); 2.92/3.44 (1H/2H, m, H-8/8'); 4.12/3.20 (1H/2H, $d / m$, J 9.5 Hz, H-9a/9'a); 3.84/3.80 (2H/2H, m/m, H-9b/9’b); 
3.89/3.84 (6H, s, MeO-3/3'); 3.82 (3H, s, MeO-4'); 5.58 $(1 \mathrm{H}, l s, \mathrm{OH}-\mathrm{C} 4)$.

${ }^{13} \mathrm{C}$ NMR (125 MHz, CD $\left.\mathrm{CN}_{3}\right), \delta: 133.9 / 131.9$ (C-1/1'); 109.9/109.8 (C-2/2'); 147.4/148.2 (C-3/3'); 145.8/149.2 (C-4/4'); 114.6/111.7 (C-5/5'); 119.1/118.0 (C-6/6'); 87.8/81.9 (C-7/7'); 54.5/50.1 (C-8/8'); 70.9/69.4 (C-9/9'); 55.9/55.5 (MeO-3/3'); 55.6 (MeO-4').

MS (70 eV), m/z (Rel. Int. \%): $\mathrm{M}^{+} 372$ (100), 156 (25), 137 (13).

\section{Acknowledgments}

The authors thank to CNPq, CAPES, FAPEAM and FAPESP for providing fellowships and financial supports.

\section{References}

1. Rodrigues, W. A.; Acta Amaz. 1980, $10,1$.

2. Souza, V. C.; Lorenzi, H.; Botânica sistemática; Instituto Plantarum: São Paulo, 2005.

3. McRae, W. D.; Towers, G. H. N.; Phytochemistry 1984, 23, 1207.

4. Ayres, D. C.; Loike, J. D.; Lignans: Chemical, Biological and Clinical Properties, University Press: Cambridge, 1990.

5. Lopes, N. P.; Ph.D. Thesis, Universidade de São Paulo, 1997.

6. Hattori, M.; Hada, S.; Kawata, Y.; Tezuka, Y.; Kikuchi, T.; Namba, T.; Chem. Pharm. Bull. 1987, 35, 3315.

7. Martins, R. C. C.; Ph.D. Thesis, Universidade de São Paulo, 2002.

8. França, V. C.; Vieira, K. V. M.; Lima, E. O.; Barbosa-Filho, J. M.; Cunha, E. V. L.; Silva, M. F.; Rev. Bras. Farmacogn. 2005, 15,326 .

9. Lopes, L. M. X.; Yoshida, M.; Gottlieb, O. R.; Phytochemistry 1984, 23, 2647.

10. Kato, M. J.; Yoshida, M.; Gottlieb, O. R.; Phytochemistry 1990, 26, 283.
11. Marques, M. O. M.; Yoshida, M.; Gottlieb, O. R.; Phytochemistry 1992, 31, 4380.

12. Suarez, M.; Bonilla, J.; Diaz, A. M. P.; Achenbach, H.; Phytochemistry 1983, 22, 609.

13. Fonseca, S. F.; Barata, L. E. S.; Rúveda, E.; Baker, P. M.; Can. J. Chem. 1979, 57, 441.

14. Shimomura, H.; Sashida, Y.; Oohara, M.; Phytochemistry 1988, $27,634$.

15. Paulo, M. Q.; M.Sc. Dissertation, Universidade Estadual de Campinas, 1983

16. Braga, A. C. H.; Zacchino, S.; Badano, H.; Sierra, M. G.; Rúveda, E. A.; Phytochemistry 1984, 23, 2025.

17. Kawanishi, K.; Uhara, Y.; Hashimoto, Y.; Phytochemistry 1982, $21,929$.

18. Agrawal, P. K.; Thakur, R. S.; Magn. Reson. Chem. 1985, 23, 389.

19. Ilda, T.; Nakano, M.; Ito, K.; Phytochemistry 1982, 21, 673.

20. Chang, W. C.; Sei, C. K.; Soon, S. H.; Bong, K. C.; Hye, J. A.; Lee, M. I.; Park, S. H.; Kim, S. K.; Plant Sci. 2002, 163, 1161.

21. Kapler, G. M.; Zhang, K.; Beverley, S. M.; Nucleic Acids Res. 1990, 18, 6399.

22. Singh, S.; Mohapatra, D. P.; Sivakurnar, R.; J. Commun. Dis. 2000, 32, 289.

23. Romoff, P.; Yoshida, M.; Cien. Cult. 1997, 49, 345.

24. Jesus, M. A.; Teixeira, A. F.; Yoshida, M.; Nunomura, S. M.; Torres, Z. E. S.; Morais, S. K. R.; Collantes, I. E.; EcoWood 2006, International Conference on Evironmentally, Edições Universidade Fernando Pessoa: Portugal, 2006.

Received: May 4, 2009

Web Release Date: June 29, 2009

FAPESP helped in meeting the publication costs of this article. 